\title{
Laparoscopic Inguinal Hernia Repair at Toyoko Hospital -A Non-inferiority Study
}

\author{
Takahiro Sasaki $^{1}$, Akiyoshi Noda ${ }^{1}$, Jin Shimada ${ }^{1}$, Kenji Nishio ${ }^{1}$, \\ Kazumi Tenjin', Nobuyoshi Miyajima ${ }^{1}$, and Takehito Otsubo ${ }^{2}$
}

(Received for Publication: December 14, 2015)

\begin{abstract}
We conducted a retrospective, single-center study to test our notion that laparoscopic transabdominal preperitoneal (TAPP) mesh repair of inguinal hernia is not inferior to open repair via anterior approach. Patient characteristics and short-term treatment outcomes were compared between 142 patients with 153 defects treated by TAPP repair between January 2010 and December 2015 and 100 patients with 104 defects treated by open anterior repair between September 2008 and December 2015. Patients in the TAPP group were significantly younger than patients in the open repair group. Operation time was significantly longer in the TAPP group at 102.8 minutes, but the blood loss volume did not differ significantly between the 2 groups. Conversion from TAPP repair to open repair was necessary for 6 patients, and this was because of adhesions found intraoperatively in all 6. Significantly fewer postoperative analgesics were used by patients in the TAPP group. Hydrocele occurred as a postoperative complication in both groups, and postoperative recurrence was observed in 2 patients in the TAPP group, but the complication rate did not differ significantly between the 2 groups. Although the TAPP procedure lengthened the repair time, the absence of a significant difference in postoperative complications confirms that TAPP repair compares favorably with open anterior repair.
\end{abstract}

\section{Key words}

Laparoscopic inguinal hernia repair, TAPP

\section{Introduction}

Laparoscopic inguinal hernia repair took hold as a reliable means of herniorrhaphy in the early 1990s, propelled by the findings of Shuthe et $\mathrm{al}^{1}$. According to data obtained through the 12th questionnaire-based survey of endoscopic surgeons conducted by the Japan Society for Endoscopic Surgery in 20142), the number of mesh-based laparoscopic transabdominal preperitoneal (TAPP) hernia repairs performed in Japan increased dramatically from 12 in 1990 to 5748 in 2013. A total of 24,065 hernia surgeries have been performed in the country since $2013,7,750$ (32\%) of which have been laparoscopic surgeries. Surgical outcomes of TAPP repair were first reported in Japan in
$1993^{3)}$, but nationwide popularization of TAPP repair was hampered by the complexity of the surgical technique and issues surrounding the cost of treatment. In the meantime, laparoscopic surgery gained acceptance for cholecystectomy and widespread acceptance for gastrectomy and colectomy. The number of laparoscopic procedures performed and the number of hospitals performing laparoscopic surgeries increased as a result. The TAPP procedure, which is the primary technique employed for laparoscopic inguinal hernia repair, was then proactively introduced.

According to the same survey, nationwide popularization was occasioned by a particular increase in medical treatment fees that occurred in 2006, and the number of laparoscopic inguinal hernia repairs per-

1 Digestive Disease Center, Toyoko Hospital, St. Marianna University School of Medicine

2 Department of Gastroenterological and General Surgery, St. Marianna University School of Medicine 
formed increased from 543 in 2006 to 5748 in 2013. TAPP repair was introduced at our hospital in January 2010, and it is currently the treatment of choice for inguinal hernia. We have assumed from the start that TAPP hernia repair is at least comparable to open repair via anterior approach, and we recently conducted a retrospective comparative single-center study to test our assumption. The study was approved by the St. Marianna University School of Medicine ethics committee (approval number: 3016).

\section{Patients and Methods}

Two groups of patients were included in the study: 142 patients (153 defects) who underwent TAPP inguinal hernia repair between January 2010 and December 2015 and 100 patients (104 defects) who underwent open inguinal anterior hernia repair between September 2008 and December 2015. TAPP repair had been offered to all patients, and open anterior repair was provided for those who had refused TAPP repair. The same clinical care map was implemented for all patients in the 2 study groups.

The TAPP repair procedure is performed as follows: The patient is placed under general anesthesia, pneumoperitoneum is established, and 3 ports are placed: a 11-mm subumbilical port for introduction of the scope (11 $\mathrm{mm}$ in diameter), a lower right abdominal port $(12 \mathrm{~mm})$, and a lower left abdominal port $(5 \mathrm{~mm})$. The peritoneum is dissected from the medial side of the medial umbilical fold to the hernial orifice. Laparoscopic coagulating shears are used to perform the dissection and then to position a Bard 3D MAX (Medicon, Inc.) or TiLENE mesh (Medical Leaders Co., Ltd.). The peritoneum is repaired from inside the peritoneal cavity. At our institution, all such TAPP repairs are performed by surgeons certified by the Japan Society for Endoscopic Surgery.

Open anterior repair is performed as follows: The patient is placed under general anesthesia, and a skin incision is made in the patient's groin. One of 3 repair methods is applied, the direct Kugel patch method, the mesh plug method, or the Marcy method, via a $5-\mathrm{cm}$ incision made in the inguinal region on the affected side.

The following clinical details were obtained from patients' records for comparison between the 2 groups: age, sex, body mass index (BMI), American Society of Anesthesiologist (ASA) performance status, hernia type (per the Japanese Hernia Society classification system), duration of the surgery, intraoperative blood loss, postoperative stay (number of days), number of times postoperative analgesics were administered, postoperative complications of Clavien-Dindo grade 2 or above, and postoperative recurrence.

\section{Statistical analyses}

Study results are shown as mean (range) values or the number of patients in each group. Betweengroup differences were analyzed by paired t-test or $\mathrm{X}^{2}$ test, as appropriate, and $P<0.05$ was considered statistically significant.

\section{Results}

Patient characteristics are shown in Table 1. Patients in the TAPP group were significantly younger than patients in the open repair group (mean age, 60.5 years vs. 66.1 years, respectively). TAPP was performed more frequently in type I-2 and type IV than open repair surgery (statistically significant, $\mathrm{P}=0.03$ and 0.04 ,respectively).There was no significant between-group difference in sex, BMI, ASA status, or the number of patients with a preoperatively identified contralateral hernia (17 vs. 11 , respectively). Single-stage, bilateral hernia repair was performed in 11 of these 17 patients in the TAPP group and in 4 of these 11 patients in the open repair group. The hernias were recurrent in 8 patients in the TAPP group and in 9 patients in the open repair group.

Short-term surgical outcomes are shown per group in Table 2. The mean duration of surgery, 102.8 minutes in the TAPP group and 67.7 minutes in the open repair group, differed significantly. In the open repair group, the direct Kugel patch method $(n=68)$, the mesh plug method $(n=26)$, and the Marcy method $(n=6)$ were applied and included the repairs performed for the 100 index hernias and 4 contralateral hernias. (These 4 contralateral hernias were repaired by the mesh plug method.) There was no significant between-group difference in intraoperative blood loss volume. The number of times postoperative analgesic drugs were administered was significantly lower in the TAPP group (mean, 0.5 times) than in the open repair group (1 time). With some deviations from the care plan in both groups, there was no significant difference in the mean postoperative stay (2.9 days in the TAPP group and 2.9 days in the open repair group).

Postoperative complications included hydrocele and hernia recurrence. Hydrocele occurred in 3 patients in the TAPP group and in 3 patients in the open repair group, and postoperative recurrence was noted 
Table 1. Clinical Characteristics of Inguinal Hernia Patients, Per Study Group

\begin{tabular}{lccc}
\hline & $\begin{array}{c}\text { TAPP group } \\
(\mathrm{n}=142)\end{array}$ & $\begin{array}{c}\text { Open repair group } \\
(\mathrm{n}=100)\end{array}$ & $P$ value \\
\hline Age (years) & 60.5 (range, $19-89)$ & 66.1 (range, $25-90)$ & 0.003 \\
Sex (M/F) & $130 / 12$ & $85 / 15$ & 0.043 \\
BMI & 22.5 (range, $15.2-33.8)$ & $22($ range, $15-28)$ & 0.10 \\
ASA PS (1/2/3/4) & $75 / 51 / 16 / 0$ & $56 / 22 / 22 / 0$ & 0.11 \\
Bilateral hernia detected preoperatively & $17\left(11^{*}\right)$ & $11\left(4^{*}\right)$ & 0.39 \\
Type of hernia** & & 6 & 0.78 \\
I-1 & 7 & 43 & 0.03 \\
I-2 & 82 & 22 & 0.17 \\
I-3 & 21 & 2 & 0.13 \\
II-1 & 10 & 11 & 0.35 \\
II-2 & 10 & 9 & 0.09 \\
II-3 & 5 & 1 & 1.00 \\
III & 1 & 1 & 0.04 \\
IV & 9 & 9 & 0.32 \\
Recurrent hernia & 8 & & \\
\hline Mean value or number of patients is shown unless otherwise indicated & & \\
\end{tabular}

Mean value or number of patients is shown unless otherwise indicated.

TAPP group=patients in whom transabdominal preperitoneal repair was performed; open repair group=patients in whom open anterior repair was performed.

$\mathrm{M}=$ male, $\mathrm{F}=$ female, BMI, body mass index, ASA PS=American Society of Anesthesiologists performance status (grades 1-4).

*Patients in whom bilateral single-stage hernia repair was performed.

**According to the Japanese Hernia Society classification system

Table 2. Short-term Surgical Outcomes, Per Study Group

\begin{tabular}{|c|c|c|c|}
\hline & $\begin{array}{c}\text { TAPP group } \\
\mathrm{n}=142\end{array}$ & $\begin{array}{l}\text { Open repair group } \\
n=100\end{array}$ & $P$ value \\
\hline Duration of surgery (min) & $102.8(42-265)$ & $67.7(26-144)$ & $<0.001$ \\
\hline Blood loss volume (mL) & $6.8(1-260)$ & $8.2(1-70)$ & 0.26 \\
\hline Postoperative hospital stay (days) & $2.9(1-11)$ & $2.9(1-11)$ & 0.43 \\
\hline Postoperative use of analgesic drugs & & & $<0.001$ \\
\hline (No. of times administered) & $0.5(0-4)$ & $1.0(0-3)$ & \\
\hline \multicolumn{4}{|l|}{ Complications } \\
\hline Hydrocele & 3 & 3 & 0.37 \\
\hline Recurrence & 2 & 1 & 0.38 \\
\hline
\end{tabular}

in 2 patients in the TAPP group and in 1 patient in the open repair group. The complication rates did not differ significantly.

Conversion from TAPP to the open repair procedure was necessary in 6 patients (Table 3). All 6 conversions were required because of abdominal adhesions. The mean duration of the conversion surgeries (including the attempted TAPP repairs) was $126 \mathrm{mi}-$ nutes (range, 60-256 minutes), and mean blood loss volume was $59 \mathrm{~mL}$. Postoperative analgesics were administered twice in 3 of these patients and not at all in the other 3 . The mean postoperative stay was 4 days.

\section{Discussion}

Heartened by the findings of important metaanalyses $^{4,5)}$, we adopted TAPP hernia repair at our hospital with enthusiasm, and we have come to appreciate the advantages of this laparoscopic repair technique. The hernial orifice can be directly confirmed during laparoscopic hernia repair, and the TAPP procedure may be indicated not only for internal and external inguinal hernias but also for femoral and suprapubic hernias. An additional benefit is that it facilitates diagnosis of contralateral, subclinical hernias ${ }^{6}$. Because TAPP repair has gradually become the procedure of choice at our hospital, we felt it necessary to confirm that the method is indeed not infe- 
Table 3. Clinical Details of Cases Requiring Conversion from TAPP to Open Anterior Repair

\begin{tabular}{|c|c|c|c|c|c|c|}
\hline & Patient 1 & Patient 2 & Patient 3 & Patient 4 & Patient 5 & Patient 6 \\
\hline Age (years) & 72 & 50 & 59 & 76 & 48 & 66 \\
\hline Sex & Female & Male & Male & Male & Male & Male \\
\hline Type of hernia & IV & $\mathrm{I}-3$ & $\mathrm{I}-3$ & $\mathrm{~V}$ & $\mathrm{I}-3$ & $\mathrm{I}-32$ \\
\hline Side & Right & Left & Left & Left & Right & Left \\
\hline Duration of surgery (min) & 60 & 121 & 120 & 75 & 256 & 126 \\
\hline Blood loss volume (mL) & 2 & 15 & 50 & 4 & 260 & 23 \\
\hline $\begin{array}{l}\text { Postoperative hospital stay } \\
\text { (days) }\end{array}$ & 5 & 3 & 3 & 4 & 4 & 5 \\
\hline $\begin{array}{l}\text { Postoperative use of analgesics } \\
\text { (No. of times administered) }\end{array}$ & 0 & 0 & 2 & 2 & 0 & 2 \\
\hline Repair method & Mesh plug & Mesh plug & Direct Kugel & Direct Kugel & Mesh plug & Direct Kugel \\
\hline Complications & None & None & None) & None & None & None \\
\hline Cause of adhesions & $\begin{array}{c}\text { Open } \\
\text { cholecystecomy }\end{array}$ & $\begin{array}{c}\text { Open } \\
\text { appendectomy }\end{array}$ & Unknown & Unknown & Unknown & $\begin{array}{c}\text { Open } \\
\text { appendectomy }\end{array}$ \\
\hline
\end{tabular}

rior to open repair, and thus we conducted the study described herein.

We found type IV hernias to be significantly more common in our TAPP repair group than in our open repair group, suggesting that the coexistence of other types of hernia that are difficult to identify intraoperatively during open anterior repair are more easily identified during TAPP repair. Not detecting coexisting hernias of different types is a factor that has been shown to increase the risk of postoperative recurrence $^{7}$, and thus we found the TAPP procedure to aid in the reduction of postoperative recurrence. We also found it possible to definitively diagnose contralateral, subclinical hernias intraoperatively during TAPP repair. These hernias were difficult to identify preoperatively, and when adequate informed consent was obtained, we were able to perform singlestage repair. Six patients in our TAPP repair group did not provide preoperative informed consent, and the fact that subclinical hernias that could be diagnosed intraoperatively were left untreated is a point that requires consideration. We believe that it is, in the very least, necessary to warn the patient to be attentive to signs of onset on the contralateral side during the postoperative follow-up period.

The longer operation time associated with TAPP repair (vs. open repair) is not unexpected and may be considered by some as a problem to be resolved. Hernia surgery is commonly considered a gateway to success for junior surgeons, and we also have a number of training options involving procedures like TAPP repair so that junior surgeons at our hospital can improve their skills. However, the clinical rotation system makes it difficult for a junior surgeon to perform enough TAPP surgeries to accumulate adequate experience, and this could prove to be an obstacle to reducing the operation time. Because retaining surgeons in one learning area has become a challenge, we believe further standardization of surgical techniques, an improved understanding of the local anatomy of the abdominal cavity, and improved peritoneal suture closure are essential if we expect to improve surgeons' basic laparoscopic skills and thus decrease the operation time.

Of note is the markedly low blood loss volumes achieved in our TAPP repair group. Large amounts of blood are seldom lost when open anterior repair is performed, and we believe that the low volumes in our TAPP group were due to the magnifying views obtained during laparoscopic surgery.

According to an early comparative investigation of TAPP vs. open surgery, postoperative stays were 5.3 days and 5.8 days, respectively ${ }^{6}$. Although the postoperative stay tended to be shorter in our TAPP repair group, we feel that the surgical outcomes in terms of postoperative stay reflect the various hernia pathologies more than they reflect the interventional methods ${ }^{6}$. Although patients in our 2 study groups were subject to the same care pathway, it was difficult to compare the outcomes in terms of the postoperative hospital stay, but we judge the mean 2.9-day postoperative stay in our TAPP group to be a very favorable outcome. We acknowledge, however, that greater efforts are needed to further shorten the hospital stay while continuing to ensure safety during the perioperative period.

A recent report of TAPP inguinal hernia repair in Japan documented a low analgesic requirement in patients who underwent the procedure ${ }^{8)}$. This low analgesic requirement held true in our TAPP group as well. The reduced need for analgesics was not reflected in our TAPP patients' hospital stay, but this is probably because the same clinical path was adhered to in both groups. Perhaps in the future it will be- 
come evident that the clinical path for TAPP patients can be adjusted.

According to the aforementioned survey ${ }^{2)}$, the main complications associated with TAPP repair are hydrocele and hematoma (82\%), gastrointestinal injury $(1.1 \%)$, and bladder injury $(0.3 \%)$. Hydrocele and hematoma occurred in 1 patient each in our TAPP group, for a combined complication rate of $2 \%$. Both complications resolved under conservative treatment, but it is vital for us to reduce the incidence of such complications through careful consideration of surgical techniques, such as management of the hernial sac. Laparoscopic repair-specific complications requiring conversion to open surgery, such as intraoperative hemorrhage that is difficult to control and postoperative bowel obstruction, must also be avoided $^{7}$. We have not encountered intraoperative hemorrhage, organ injury, or any serious intraoperative complication that required conversion to laparotomy, and we believe that the participation of a surgeon certified by the Japan Society for Endoscopic Surgery was a key factor in avoidance of these issues.

The reported post-inguinal hernia repair recurrence rate is $4 \%$; mesh migration (7.2\%), inappropriate mesh size $(2.9 \%)$, and suprapubic hernia (2.1\%) are also reported ${ }^{2}$. We encountered swelling on the day after surgery in 2 of our patients $(1.3 \%)$, both treated during the early period after introduction of TAPP hernia repair at our hospital. In both cases, laparoscopic examination at the time of repeat surgery showed that the mesh had rolled up, and we performed open anterior repair (with a mesh plug). We have learned that the size of the mesh should be determined on the basis of the surface area of dissection. During the early period, we fixed the mesh only at 2 points along Cooper's ligament, but we now fix the mesh at 5 or 6 points, including the rectus abdominis muscle lateral to the inferior epigastric vessels, and we have since encountered no recurrence.

The unexpected need for conversion to open anterior repair is another matter to be addressed. As noted above, adhesions necessitated such conversion in 6 of our patients. Of note is the fact that only 3 of these patients had undergone a prior abdominal surgery. In 1 of the 6 patients (Patient 5), severe bleeding occurred during the detachment, resulting in a blood loss volume of $260 \mathrm{~mL}$ and a 256-minute operation (including the attempted TAPP repair). The high blood loss volume in this particular patient raised the mean volume to $59 \mathrm{~mL}$ for our 6 patients who underwent conversion to open anterior repair, but the mean volume for the 5 other patients was $18.8 \mathrm{~mL}$, which is clinically acceptable.

The initial attempt to perform TAPP repair in the 6 patients who required conversion to open surgery provided us with a unique opportunity to observe the herniotomy and repair from within the abdominal cavity. We were able, for example, to confirm precise placement of the mesh.

The adhesions that necessitated conversion surgery resulted from a prior open appendectomy in 2 patients and open cholecystectomy in 1. Laparoscopic adhesiotomy can be difficult and is sometimes impossible; there is a risk of peritoneal injury in some cases. Adhesions can choke the presumptive hernia repair region. In the other 3 patients who required conversion surgery, similar adhesions were unexpectedly found, but the source of these adhesions is unknown. These patients had not undergone prior abdominal surgery. This highlights the need for surgeons to be aware of the possibility of demoplasia in any patient requiring inguinal hernia repair.

\section{Conclusion}

The short-term outcomes of TAPP hernia repair performed in our department have been good. Although the operation time has been longer than that of open surgery, it has equaled the time that is currently considered generally acceptable. We found the reported decreased need for postoperative analgesics to be a true advantage. One caveat is that, in attempting TAPP repair, surgeons must remain vigilant and prepared for conversion to open surgery should severe adhesions be encountered. Going forward, we believe that an accumulation of cases will allow us to thoroughly standardize the TAPP procedure, which will in turn decrease the duration of surgery and the recurrence rate.

\section{References}

1) Schultz L, Graber J, Piertrafitta J, Hickok D. Laser laparoscopic herniorrhapy: a clinical trial preliminary results. J Laparoendosc Surg 1990; 1: 41-45.

2) Journal of Japan Society for Endoscopic Surgery. $12^{\text {th }}$ Nationwide Survey of Endoscopic Surgery in Japan.2014; 19: 520-524.

3) Matsumoto $S$, Kawabe N, Mori K, Suzuki K, Miyata S, Yasaka O, Oshima R, Kobayashi K, Matsumoto K, Yoshida Y, Itonano T, Kimura T, Nagai K, Inmaki T, Yunaya T. Experience of laparoscopic inguinal hernioplasty [in Japanese 
with English abstract]. Jpn J Gastroenterol Surg 1993; 26: 2329-2432.

4) EU Hernia Trialists Collaboration. Laparoscopic compared with open methods of groin hernia repair: systematic review of randomized controlled trials. Br J Surg 2000; 87: 860-867.

5) Schmedt CG, Sauerland S, Bittner R. Comparison of endoscopic procedures vs Lichtenstein and other open mesh techniques for inguinal hernia repair: a meta-analysis of randomized controlled trials. Surg Endosc 2005; 19: 188199.

6) Yamamoto A, Fujimura M, Hirano M, Subou M, Okada J, Mori A, Tanaka H, Sato I, Soeda Y. Experience of laparoscopic hernioplasty. Nihon Rinsho Geka Gakkai Zasshi (J Jpn Surg Assoc)
1994; 55: 1974-1979.

7) Kimura T, Yoshida M, Kobayashi T, Sakuramachi S, Yoshino A, Takabayashi N, Wada H, Suzuki K, Ishihara N, Takeuchi Y, Suzuki K, Nakamura M, Harada Y. Special issue: reinvestigation of inguinal hernia. Laparoscopic hernia repair-2-indications and limitations. Surgical Diagnosis \& Treatment 1995; 45: $437-$ 443.

8) Ozaki Y, Shibata S, Ueta T, Yamashiro Y, Yamaguchi $\mathrm{Y}$, Ishiguro $\mathrm{M}$, Nishidoi $\mathrm{H}$, Tatebe $\mathrm{S}$. The initial results of laparoscopic inguinal hernioplasty (transabdominal preperitoneal approach: TAPP). The Journal of the Tottori Medical Association 2015; 43: 11-14. 